\title{
MEMORY
}

\section{Resistive RAM arrays with multiple bits}

IEEE Trans. Electron Devices 66, 641-646 (2019)

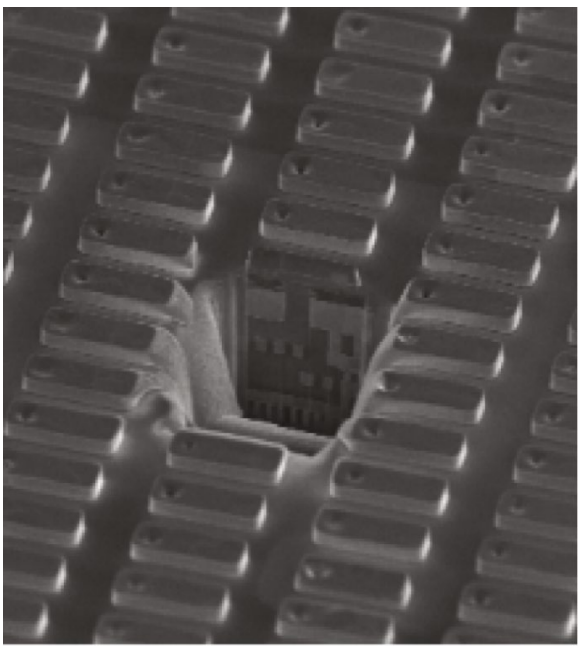

Credit: IEEE

Resistive random access memory (RRAM), which has a switching mechanism based on conducting filaments, is a promising non-volatile storage technology. To achieve high-density RRAM, one approach is to use multilevel cell storage, which allows more than one bit to be stored in a single cell. In this setup, the resistance window of a cell is divided into different ranges corresponding to different bits. However, variations in the resistances of the cells, which is related to variations in the compositions of the conductive filaments, makes reliable programming of multiple RRAM cells a challenging task. Binh Le and colleagues have now demonstrated efficient programming of arrays of RRAM in which each cell stores three bits.

The researchers - who are based at Stanford University and CEA-Leti in Grenoble - developed a programming scheme that takes the variations in resistance values into account in order to allocate resistance ranges instead of dividing them equally. The approach also minimizes the gaps between the resistance ranges (which are required for the read operation). This non-uniform distribution of ranges increases the probability of programming the cells to the correct range with less iteration. To test the technique, Le and colleagues used $4 \mathrm{kbit}$ arrays of hafnium oxide-based RRAM cells, which were fabricated with $130 \mathrm{~nm}$ silicon complementary metal-oxide-semiconductor (CMOS) technology. They found that, after 4,000 cycles, the number of iterations needed to program the cells was 11 , whereas more than 20 iterations were needed with the method of allocating the ranges uniformly.

Christiana Varnava

Published online: 15 February 2019

https://doi.org/10.1038/s41928-019-0218-1 